Received 20th August 2018 Accepted 10th October 2018

DOI: $10.1039 / \mathrm{c} 8 \mathrm{sm} 01714 \mathrm{j}$

rsc.li/soft-matter-journal

\section{Carbon dot aggregates as an alternative to gold nanoparticles for the laser-induced opening of microchamber arrays $\dagger$}

Olga A. Sindeeva, (D)*ab Ekaterina S. Prikhozhdenko, (D) ${ }^{a}$ Daniil N. Bratashov, (D) ${ }^{a}$ Anna M. Vostrikova, ${ }^{a}$ Vsevolod S. Atkin, ${ }^{a}$ Aleksey V. Ermakov, ${ }^{a}$ Boris N. Khlebtsov, ${ }^{\text {ac }}$ Andrei V. Sapelkin, ${ }^{a b}$ Irina Yu. Goryacheva (D) ${ }^{a}$ and Gleb B. Sukhorukov*ab

Carbon dots (CDs) are usually used as an alternative to other fluorescent nanoparticles. Apart from fluorescence, CDs also have other important properties for use in composite materials, first of all their ability to absorb light energy and convert it into heat. In our work, for the first time, CDs have been proposed as an alternative to gold nanostructures for harvesting light energy, which results in the opening of polymer-based containers with biologically active compounds. In this paper, we propose a method for the synthesis of polylactic acid microchamber arrays with embedded CDs. A comparative analysis was made of the damage to microchambers functionalized with gold nanorods and with CD aggregates, depending on the wavelength and power of the laser used. The release of fluorescent cargo from the microchamber arrays with $C D$ aggregates under laser exposure was demonstrated.

\section{Introduction}

Toxicity remains an issue with many modern drugs, causing an undying interest in controlled delivery systems, which make the deployment of drugs local and timely and that reduce side effects. The microchamber array (MCA) is a recently introduced promising drug delivery system, ${ }^{1-5}$ allowing encapsulation of active components ${ }^{6,7}$ to stimulate individual cells through locally released cargo ${ }^{8}$ and of nanoparticles ${ }^{9}$ ensuring sensitivity to external stimuli. ${ }^{7,10,11}$ The unique properties of polylactic acid (PLA), such as outstanding barrier properties, ${ }^{12}$ biocom40 patibility, and biodegradability, ${ }^{13,14}$ make it a good candidate for use in MCA design.

Site- and time-specific controlled drug release is especially important for various applications. Therefore, the past few decades have seen an active interest in ways of triggering drug far, external stimuli such as the electromagnetic field, ${ }^{16,17}$ ultrasound, ${ }^{18,19}$ microwave radiation, ${ }^{20}$ and laser exposure ${ }^{21,22}$ have been used for remote triggering. Laser opening is a well-

$50{ }^{a}$ Saratov State University, Saratov, 410012, Russia.

E-mail: o.a.sindeeva@gmail.com

${ }^{b}$ School of Engineering and Materials Science, Queen Mary University of London, Mile End Road, London, E1 4NS, UK. E-mail: g.sukhorukov@qmul.ac.uk

${ }^{c}$ Institute of Biochemistry and Physiology of Plants and Microorganisms RAS, Saratov, 410049, Russia

$55 \dagger$ Electronic supplementary information (ESI) available. See DOI: 10.1039/ c8sm01714j established and widely used method in targeted drug delivery owing to its precise beam focusing, good tissue penetration ability, and safety for living cells [for near-infrared (NIR) light].

For controlled drug release triggered with laser exposure, photoabsorption agents are usually employed. These agents convert photon energy into heat, and this is followed by the local destruction of some thermosensitive materials and by drug release from the carriers. Inorganic nanomaterials such as gold nanoparticles (GNPs), ${ }^{23,24}$ carbon nanotubes, ${ }^{25,26}$ graphene, ${ }^{27}$ copper sulfide nanoparticles (NPs), ${ }^{28}$ palladium nanosheets, ${ }^{29}$ and organic nanomaterials ${ }^{30,31}$ usually function as photoabsorption agents.

Undoubtedly, the most well-known, well-proven, and widely used thermosensitive material for carrier opening in vitro and in vivo is gold nanoparticles (GNPs). ${ }^{32-37}$ However, the current situation with GNP cytotoxicity and biocompatibility remains controversial. Some authors argue, for instance, that particle toxicity depends on the dose and the administration route used. ${ }^{38}$ Others are sure that the use of GNPs is $\operatorname{safe}^{36,39}$ and that the manifested toxicity is due to the ligands used to stabilize the particles. ${ }^{37,40}$ However, all authors agree that GNPs are not biodegradable ${ }^{41-43}$ unlike carbon dots. ${ }^{44}$ Thus, the biomedical application of such agents is complicated.

This work aims at introducing a more affordable, biocompatible, and biodegradable material for targeted drug delivery systems, which is sensitive to laser exposure. We report a proofof-concept study to substitute carbon nanostructures for GNPbased materials. 


\section{Experimental section}

\subsection{Materials}

For MCA and carbon dot (CD) synthesis, PLA (3 mm granule, $M_{\mathrm{w}} \sim 60000$ ), chloroform, Nile red, fluorescein isothiocyanatedextran (FITC-dextran, $M_{\mathrm{w}} \sim 70000$ ), and dextran sulfate sodium salt (DSS, $M_{\mathrm{w}} \sim 40 \mathrm{kDa}$ ) were all purchased from Sigma-Aldrich. The poly(dimethylsiloxane) (PDMS) kit, consisting of a prepolymer and a curing agent (Sylgard 184), was purchased from Dow-Corning, Midland, USA.

For the gold nanorod (GNR) synthesis, all chemicals were obtained from commercial suppliers and were used without further purification. Cetyltrimethylammonium bromide (CTAB, $>98.0 \%$ ), L-ascorbic acid (>99.9\%), hydrochloric acid ( $\mathrm{HCl}, 37$ wt\% in water), and sodium borohydride $\left(\mathrm{NaBH}_{4}, 99 \%\right)$ were purchased from Sigma-Aldrich. Hydrogen tetrachloroaurate trihydrate $\left(\mathrm{HAuCl}_{4} \cdot 3 \mathrm{H}_{2} \mathrm{O}\right)$ and silver nitrate $\left(\mathrm{AgNO}_{3},>99 \%\right)$ were purchased from Alfa Aesar. Ultrapure water obtained from a Milli-Q Integral 5 system with a resistivity of $\sim 18.2 \mathrm{M} \Omega \mathrm{cm}$ was used in all experiments.

\subsection{Synthesis of gold nanorods}

GNRs were fabricated by the seed-mediated method ${ }^{45}$ with minor modifications. ${ }^{46}$ In the first step, a seed suspension was obtained by mixing $10 \mathrm{~mL}$ of $0.1 \mathrm{M} \mathrm{CTAB}$ and $250 \mu \mathrm{L}$ of $10 \mathrm{mM} \mathrm{HAuCl}_{4}$, followed by adding $1 \mathrm{~mL}$ of ice-cold $10 \mathrm{mM}$ $\mathrm{NaBH}_{4}$. In the second step, GNRs were obtained by mixing 900 $\mu \mathrm{L}$ of $0.1 \mathrm{M} \mathrm{CTAB}, 50 \mathrm{~mL}$ of $10 \mathrm{mM} \mathrm{HAuCl}_{4}, 20 \mathrm{~mL}$ of $4 \mathrm{mM}$ $\mathrm{AgNO}_{3}, 10 \mathrm{~mL}$ of $0.1 \mathrm{M}$ ascorbic acid, $10 \mathrm{~mL}$ of $1 \mathrm{M} \mathrm{HCl}$, and 10 $\mathrm{mL}$ of the seed suspension. As-prepared nanorods were centrifuged at $12000 \mathrm{~g}$ for $60 \mathrm{~min}$, and the pellet was resuspended in water. The final concentration of GNRs was about $10^{12}$ particles per $\mathrm{mL}$. Transmission electron microscopy (TEM) analysis showed that the average width and length of the GNRs were $11 \pm 3 \mathrm{~nm}$ and $40 \pm 6 \mathrm{~nm}$, respectively. The longitudinal resonance was located near $790 \mathrm{~nm}$, corresponding to an axial ratio of about 3.8 .

\subsection{Synthesis of carbon dots} esis in doubly distilled water. A DSS water solution (concentration, $10 \mathrm{mg} \mathrm{mL}^{-1}$ ) was made that was transferred to a glass cup, placed into a Teflon cup with a tight-fitting cover, put into a stainless-steel autoclave, and heated at $200{ }^{\circ} \mathrm{C}$ for $3 \mathrm{~h}$. The resulting solution was cooled to room temperature. Finally, the solution was centrifuged $(7500 \mathrm{~g}, 20 \mathrm{~min})$ and the supernatant liquid was collected. The spectrum of the carbon dots was presented in our previous work ${ }^{47}$ and is included in the ESI. $\dagger$

\subsection{Fabrication and functionalization of PLA microchamber} arrays

For the fabrication of PLA MCAs, a patterned PDMS stamp was prepared. Stamps with microwells were prepared from a prepolymer-curing agent $(10: 1)$ mixture. Silicon masters with 190000 micro-pillars were immersed into this mixture and degassed for $30 \mathrm{~min}$ in a vacuum. PDMS solidified at $70{ }^{\circ} \mathrm{C}$ within $3 \mathrm{~h}$, after which it was cut out and separated from the silicon masters. The silicon masters had been prepared by traditional photolithography and etching at Shenzhen Semiconductor, Shenzhen, China, and had round micropillars with a diameter of $10 \mu \mathrm{m}$, a height of $5 \mu \mathrm{m}$, and a center-to-center distance of $20 \mu \mathrm{m}$.

The PLA MCAs were made up of two parts: a patterned film and a flat film. For making a patterned PLA film with microwells, the PDMS stamp was dip-coated with a 1 wt\% PLA solution in chloroform for $5 \mathrm{~s}$. The patterned PLA microfilm on the PDMS stamp was printed onto the flat PLA microfilm on cover glass under pressure $\left(2 \mathrm{~kg} \mathrm{~cm}{ }^{-2}\right)$ for $60 \mathrm{~s}^{7}$ After printing, the PDMS stamp was removed.

For the laser-induced opening of microchambers, the patterned film was modified with GNRs and CD aggregates (CDAs). GNRs were sedimented onto the inner surface of the patterned film. CDAs were obtained by dispersing dry CDs in a PLA solution in chloroform.

\subsection{Laser-induced opening of microchamber arrays}

This was carried out with a Renishaw inVia confocal Raman microscope (Renishaw, UK) equipped with $785 \mathrm{~nm}$ and $532 \mathrm{~nm}$ lasers. Laser power for both lasers was controlled by neutral optical density filters. The laser beam was focused through a $50 \times$ (Leica N PLAN L, N.A. 0.5) microscope objective. With both lasers, the laser powers were 100,50 , and $10 \%$, corresponding to $1.662 \times 10^{4} \mathrm{~W} \mathrm{~cm}^{-2}, 0.831 \times 10^{4} \mathrm{~W} \mathrm{~cm}^{-2}$, and $0.166 \times 10^{4} \mathrm{~W}$ $\mathrm{cm}^{-2}$ for the $532 \mathrm{~nm}$ laser and to $0.804 \times 10^{4} \mathrm{~W} \mathrm{~cm}^{-2}, 0.402 \times$ $10^{4} \mathrm{~W} \mathrm{~cm}^{-2}$, and $0.201 \times 10^{4} \mathrm{~W} \mathrm{~cm}^{-2}$ for the $785 \mathrm{~nm}$ laser. The laser exposure time was $1 \mathrm{~s}$ in all experimental series.

\subsection{Study of controlled fluorescent dye release from single microchambers}

For the study of fluorescent cargo release, the MCA shell was labelled with Nile red dye (concentration, $100 \mu \mathrm{g} \mathrm{mL}^{-1}$ ), which had been added to the PLA solution before MCA preparation. The microchambers were filled with a water solution of FITCdextran (concentration, $10 \mathrm{mg} \mathrm{mL}^{-1}$ ). The samples were then dried, sealed, and placed in a $2 \%$ agarose gel to reduce the fast diffusion of the fluorescent cargo and to detect slow release.

Before and after laser exposure, the MCAs were analyzed with a Leica TCS SP8 X laser scanning confocal microscope (Leica, Germany). The excitation laser lines for FITC-dextran and Nile red were 495 and $552 \mathrm{~nm}$, respectively. Images and 3D scans were recorded by using two fluorescence channels: 505$540 \mathrm{~nm}$, corresponding to the fluorescence of FITC, and 565$619 \mathrm{~nm}$, corresponding to the fluorescence of Nile red. Optical images of the samples were also recorded. The z-step between the confocal planes was $1 \mu \mathrm{m}$. 3D reconstruction of the region of interest was done with Leica LAS X software.

\subsection{Characterization techniques}

Scanning electron microscopy (SEM) images of MCAs before and after laser exposure were obtained with a Tescan MIRA II LMU setup (Tescan, Brno, Czech Republic). Samples were coated with an approximately $5 \mathrm{~nm}$-thick gold film by using 
1 an Emitech K350 sputter coater (Quorum Technologies Ltd, Ashford, UK). Images were collected at a $5 \mathrm{kV}$ accelerating voltage to avoid beam-induced damage to the soft polymer samples.

TEM images of MCAs with GNRs were obtained with a Libra120 transmission electron microscope (Carl Zeiss, Germany) operating at $120 \mathrm{kV}$. GNRs were deposited on a 300 mesh copper grid coated with formvar. GNR diameters and lengths were evaluated from digitized TEM images (Grapher 8, Golden Software, Inc.) of about 300 GNRs.

A scanning confocal microscope with a $405 \mathrm{~nm}$ laser was used to visualize and characterize CDAs in PLA MCAs. 2D images were recorded by using a spectral band of 420$650 \mathrm{~nm}$, corresponding to the most intense CD fluorescence signal for the $405 \mathrm{~nm}$ excitation laser line.

The amount of GNRs in the water suspension was estimated with a Perkin-Elmer LS 55 spectrometer.

\section{Results and discussion}

3.1. Fabrication and characterization of PLA microchamber arrays with gold nanorods and carbon dot aggregates

MCAs were made by printing flat and patterned films onto each other (Fig. 1). Films were obtained by single-entry dipping of the cover glass and the patterned PDMS stamp with microwells (height, $4 \mu \mathrm{m}$; diameter, $10 \mu \mathrm{m}$; and center-to-center distance, $25 \mu \mathrm{m}$ ) into a $1 \mathrm{wt} \%$ PLA solution for $5 \mathrm{~s}$. The stamp was removed after printing. The MCAs with sealed PLA-based microchambers remained on the cover glass. GNRs (in the classical method) or CDAs (in the new method) were included in the MCA composition for the controlled opening of the microchambers with laser irradiation.

GNRs were deposited on the inner surface of the patterned microfilm from the water suspension (Fig. 1A). For this purpose, $200 \mu \mathrm{L}$ of a GNP suspension (200 $\mathrm{mg} \mathrm{mL}^{-1}$ ) was applied to a horizontally oriented film with microwells for $3 \mathrm{~h}$. Then, the drop was removed with a micropipette. Some GNRs remained

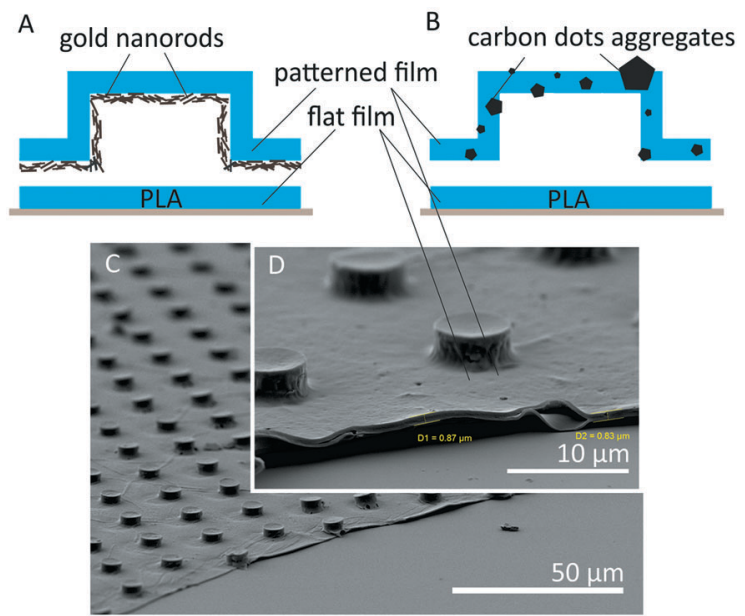

Fig. 1 Scheme of the GNR (A) and CDA (B) location in PLA MCAs. Typical SEM images of a PLA MCA with GNRs (C) and CDAs (D). on the patterned film, as shown in Fig. 2A (SEM image) and 2C (TEM image). For precipitating the GNRs, $200 \mu \mathrm{L}$ of a $0.5 \mathrm{M}$ $\mathrm{NaCl}$ solution was added to $200 \mu \mathrm{L}$ of the nanoparticle suspension. $^{48}$ The resulting suspension was centrifuged at $12000 g$, after which the supernatant liquid was removed and the GNRs were resuspended in $200 \mu \mathrm{L}$ of deionized water. As a result, more prominent aggregates of GNRs (in comparison with the non-aggregated GNRs) began to be adsorbed on the hydrophobic PLA surface, because an increase in the particle size and mass leads to an increase in the sedimentation rate. ${ }^{49}$ The GNR amount on the patterned film was estimated from the change in GNR suspension absorption before and after deposition, and it was found to be $-0.47 \mathrm{pg} \mu \mathrm{m}^{-2}$. The patterned PLA film with GNRs was printed on a flat PLA film.

For CD-based DSS, the protocol of nanoparticle inclusion in the MCAs was changed. The reason was the strong wettability of the hydrophobic surface by the CD water suspension, the long period of drying, and the associated deformation of the thin PLA film surface. Therefore, a CD water suspension was dried at $70{ }^{\circ} \mathrm{C}$, then ethanol was added, and the mixture was dried again (repeated 3 times for complete water removal). In the next step, $200 \mathrm{mg}$ of dry CDs was resuspended in $5 \mathrm{~mL}$ of $1 \mathrm{wt} \%$ PLA chloroform solution for $30 \mathrm{~min}$ by using ultrasound at $37 \mathrm{kHz}$ with an intensity of $0.087 \mathrm{~W} \mathrm{~cm} \mathrm{~cm}^{-2}$ (Elmasonic 15H; Elma Schmidbauer GmbH, Singen, Germany). As a result, we obtained large (several $\mathrm{nm}$ to $4-5 \mu \mathrm{m}$ ) CD aggregates in the PLA solution. The PDMS stamp was dipped into this suspension, and the patterned film with CDAs was then printed on a flat PLA film (Fig. 1B).

Fig. 2 shows the chaotic arrangement of CDAs on the inside (D) and outside (E) surfaces of the patterned film. The aggregates could also be visualized by confocal microscopy (Fig. 2F, $405 \mathrm{~nm}$ laser). The CD amount in the patterned film (0.37 pg $\mu \mathrm{m}^{-2}$ ) was calculated from the film thickness and the ratio of CDs to PLA. In principle, the aggregation of GNRs and CDs is assumed to have a positive effect. GNP aggregates are more sensitive to laser exposure and provide more efficient release of cargo from carriers than do non-aggregated GNPs. ${ }^{50}$ Importantly, the large CDAs obtained from DSS after dispersion in the hydrophilic medium disaggregated again, forming a finely dispersed sediment-free suspension. This property is an undoubted advantage over the not-disintegrating and nonbiodegradable GNPs in vivo. ${ }^{41-43}$

\subsection{Bubbles as a reliable marker of the opening of individual} microchambers by using a $532 \mathrm{~nm}$ and a $785 \mathrm{~nm}$ laser

Individual microchambers in the array with GNRs and CDAs were opened by exposure to a $532 \mathrm{~nm}$ and a $785 \mathrm{~nm}$ continuous wave laser, because the GNRs used have two absorption peaks in the green $(\max =511 \mathrm{~nm})$ and infrared $(\max =832 \mathrm{~nm})$ spectrum regions. The exposure time was $1 \mathrm{~s}$, and the laser powers were $1.662 \times 10^{4}$ and $0.804 \times 10^{4} \mathrm{~W} \mathrm{~cm}^{-2}$, respectively. In this experiment, the MCAs were immersed in distilled water. The patterned arrangement of the arrays and the special labeling made it possible to find the same sample region after exposure and evaluate morphological changes. 


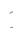

before laser exposure

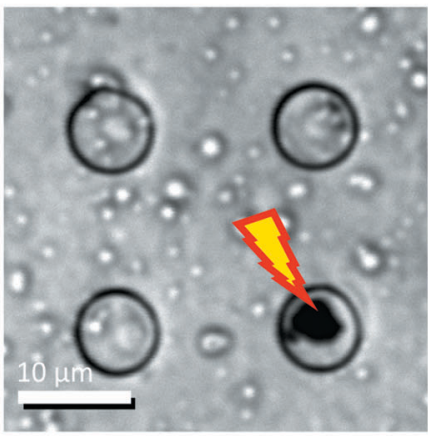

transmission
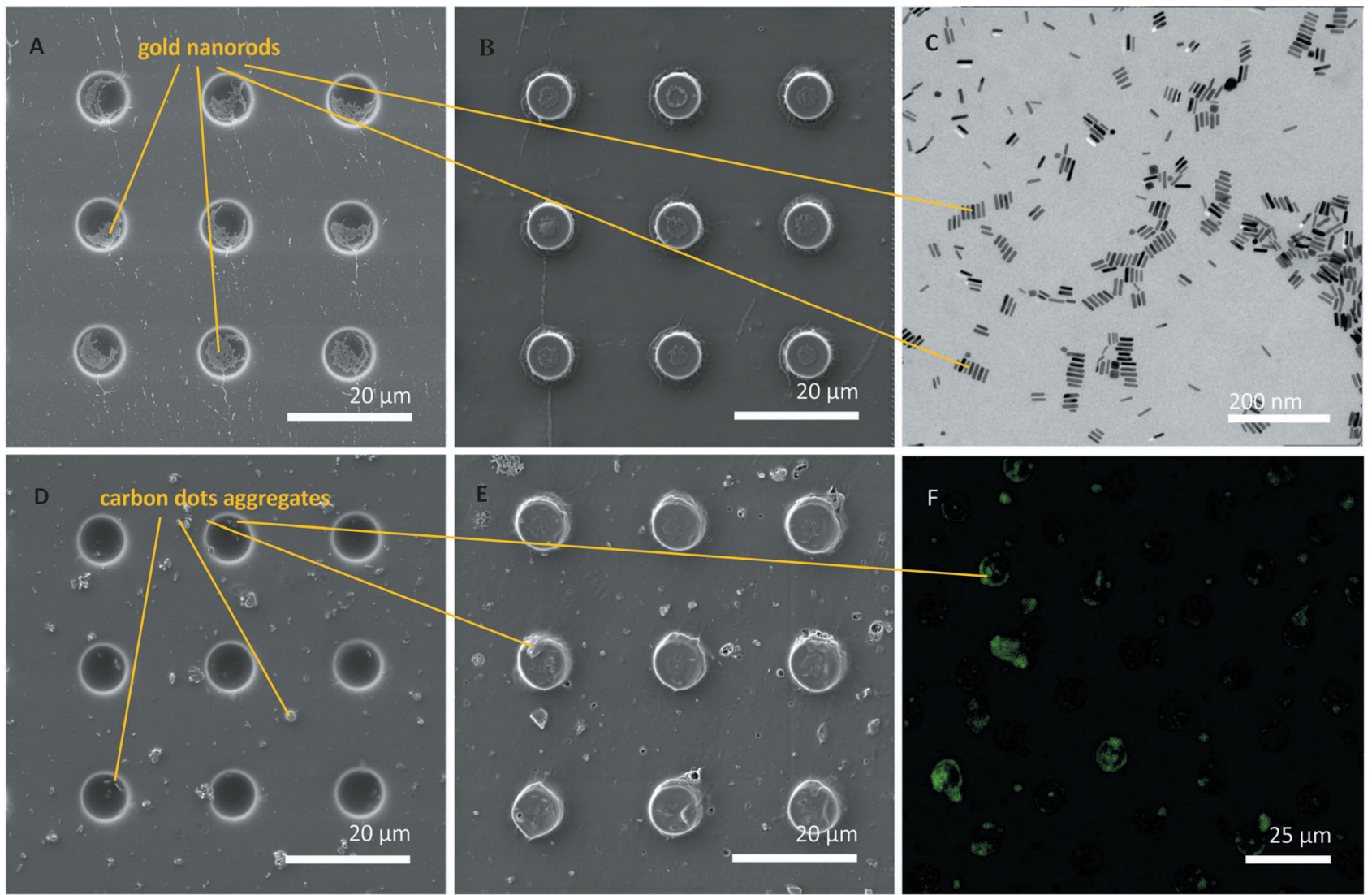

Fig. 2 SEM images of the PLA patterned film with GNRs [from inside (A) and outside (B)] and CD aggregates [from inside (D) and outside (E)]. The arrangement of GNRs (C, TEM image) and CD aggregates (F, confocal image) in the MCAs.
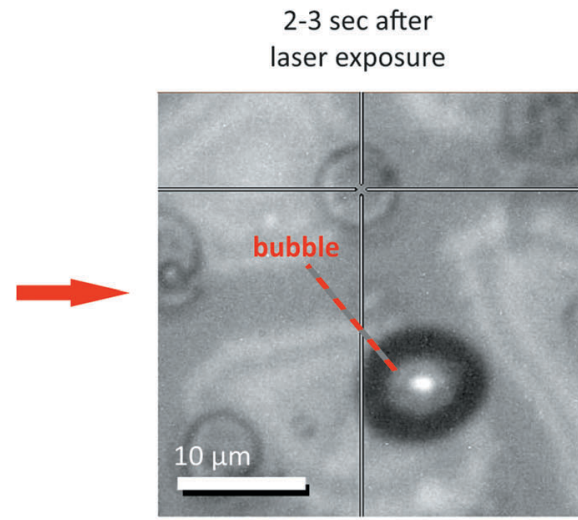

reflection
5 min after laser exposure
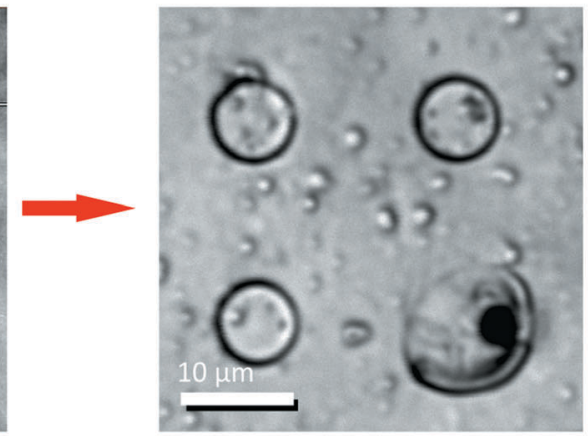

transmission
1

10

Fig. 3 Typical images of microchambers before and after $532 \mathrm{~nm}$ laser exposure (bright-field microscopy).
A reliable marker of the microchamber opening was the bubbles were observed for 2-3 s, after which they collapsed. Morphological changes in the microchambers after bubble collapse were also clearly visible in the bright-field (Fig. 3) and SEM images (Fig. 4). Bubble formation was observed both in samples with GNRs and in samples with CDAs after $532 \mathrm{~nm}$ and $785 \mathrm{~nm}$ laser exposure.
It is well known that bubble formation is associated with the boiling of a liquid by a high-power laser. ${ }^{51,52}$ This process is characterized by fast plasma formation after liquid evaporation and subsequent vapor expansion accompanied by shock wave emission. ${ }^{52}$ Bubble formation on the GNP surface irradiated by a high-power laser in water was studied for medical applications such as cancer photothermal therapy. ${ }^{53,54}$ Link and coworkers also demonstrated that two pulse lasers (nanosecond 
PLA + GNRs

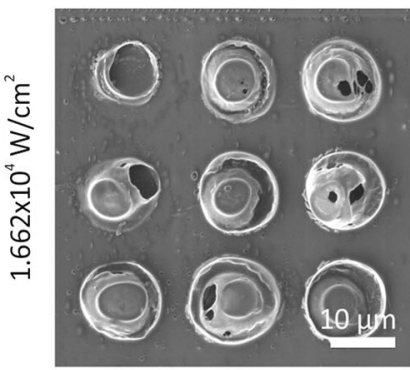

10

20

\section{Q6
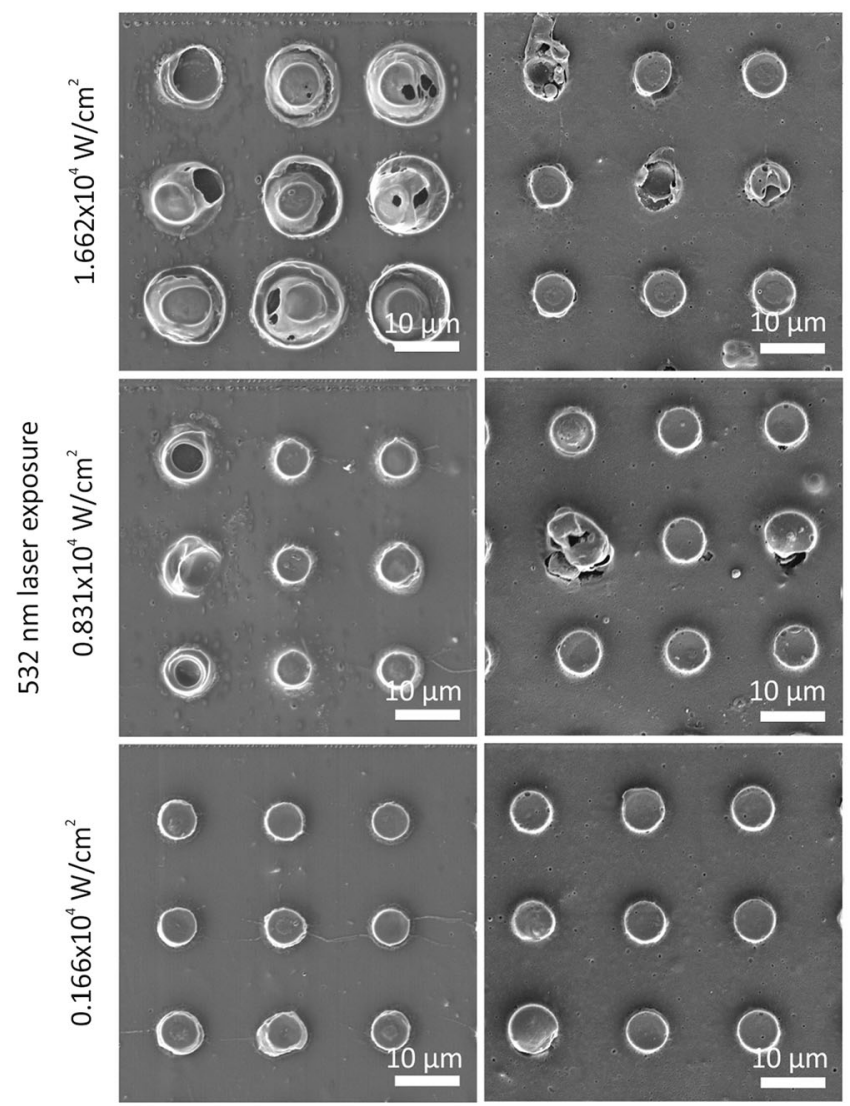

PLA + GNRs
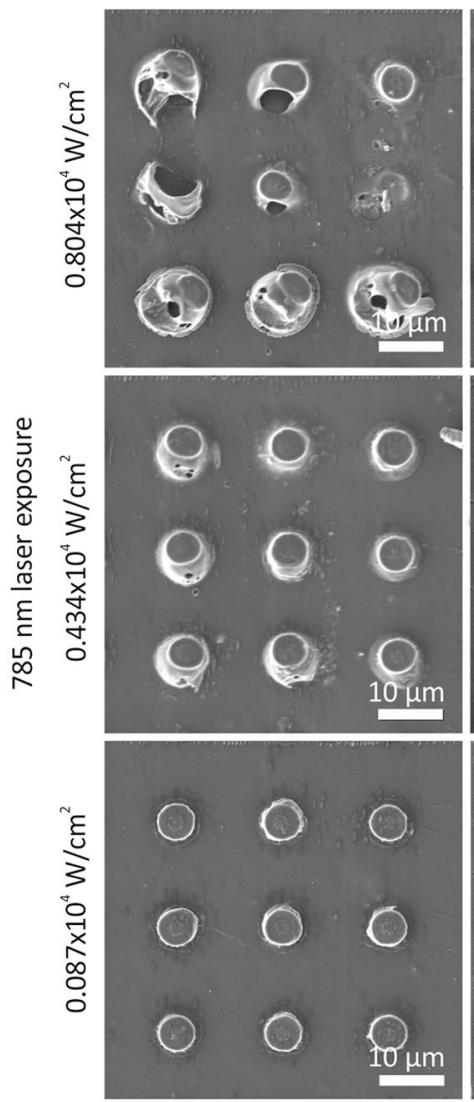

$\mathrm{PLA}+\mathrm{CDAs}$
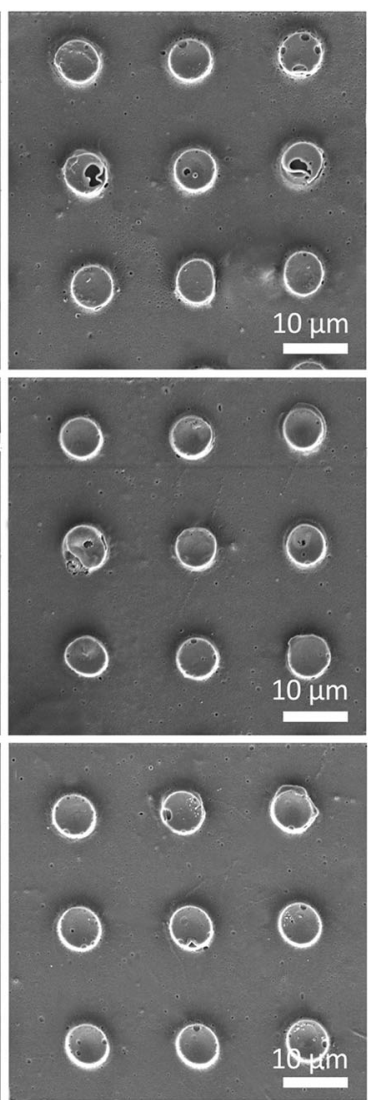

Fig. 4 Dependence of the morphological changes in the microchambers with GNRs and CDAs on the laser power and wavelength.

at $355 \mathrm{~nm}$ and femtosecond at $800 \mathrm{~nm}$ ) caused a photothermal reshaping of GNRs in an aqueous suspension. ${ }^{55,56}$ Similar data

were obtained by Chang et al., who used nanosecond lasers (532 and $1064 \mathrm{~nm}) .{ }^{55}$ Both green and infrared lasers are suitable for heating up GNRs in the polymer shell, because GNRs absorb laser energy in these regions well. ${ }^{57}$

Heating of photoabsorption agents by laser irradiation leads not only to fluid boiling but also to carrier-wall destruction. The

40 melting of the walls of carriers with GNPs and with carbon nanotubes under laser exposure has been well studied. ${ }^{32-35,50}$ The destruction of the microchamber walls is not directly related to bubble formation, but it is a reliable marker of sufficient heating up of photoabsorption agents for the open-

45 ing of the microchambers under laser exposure. Note that other authors also recorded a relationship between damage to polymeric shells with GNPs and laser power. ${ }^{32}$

\subsection{Morphological changes in microchamber structure, depending on the wavelength and power of the laser used}

A detailed study of the morphological changes in microchamber structure showed that with increasing laser power (532 and $785 \mathrm{~nm}$ ), the damage to the PLA shell with GNRs and CDAs became greater (Fig. 4).

In the microchambers without large aggregates (smaller than 2-4 $\mu \mathrm{m}$ ), we did not notice any changes after exposure, either by optical or by scanning electron microscopy. Of note, in both samples (with GNPs and CDAs), the changes were more pronounced after the $532 \mathrm{~nm}$ laser was used, possibly owing to the higher power density. With the same laser power and the same objective lens, the laser power density was nearly two times higher for the $532 \mathrm{~nm}$ laser than it was for the $785 \mathrm{~nm}$ laser.

Traditionally, GNPs are used for the controlled release of encapsulated materials from polymeric microcapsules. ${ }^{32,58,59}$ The principle of shell opening is based on the interaction of the laser energy source with GNPs, which absorb laser energy and heat up. The heating results in a rupture of the polymer shell and a release of the encapsulated materials. ${ }^{50}$ GNP exposure to a laser results in a high local gradient of heat and in moderate heating of the whole volume of the solution around the carriers' polymeric shells. ${ }^{60}$ If the GNPs are located in close proximity to each other, the heat emission processes add up. However, our research showed that the microchambers could also be opened by heating the CDAs. In this case, the SEM images show that the damage to the microchambers was more local in comparison with the use of GNRs, because CDAs were not evenly distributed. Moreover, the use of materials based on CDs has a number of other advantages. CDs are generally considered as an alternative to conventional organic dyes, and they also have significant advantageous characteristics such as high 
1 photostability and tunable emission. ${ }^{61}$ CDs have excellent water solubility ${ }^{62}$ and are more environmentally friendly and much safer for biological use than most traditional quantum dots and heavy metal ions. ${ }^{63,64}$ It was shown that after intravenous injection, $35 \mathrm{~nm}$ CDs accumulate in the reticuloendothelial system and kidney, and then they are gradually excreted both renally and fecally. A dose of $20 \mathrm{mg} \mathrm{kg}^{-1}$ appears safe to use in experimental animals over a period of 3

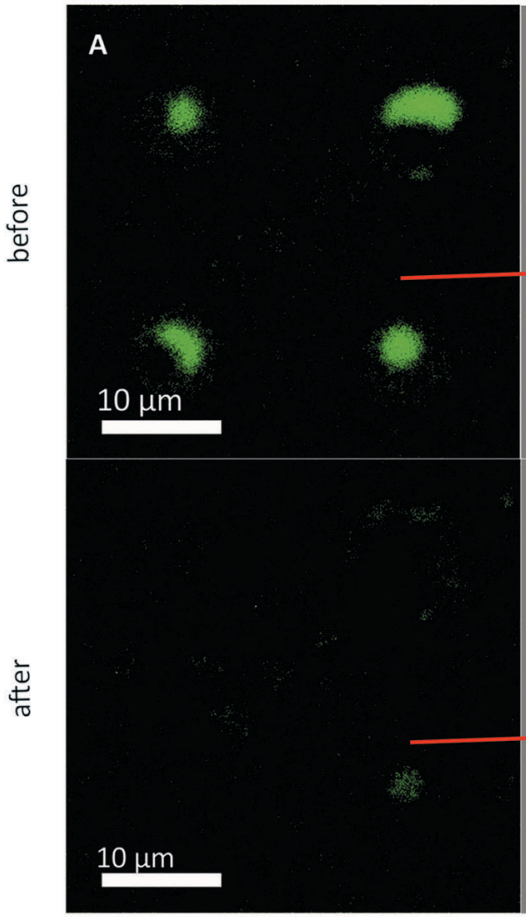

$532 \mathrm{~nm}$ laser exposure

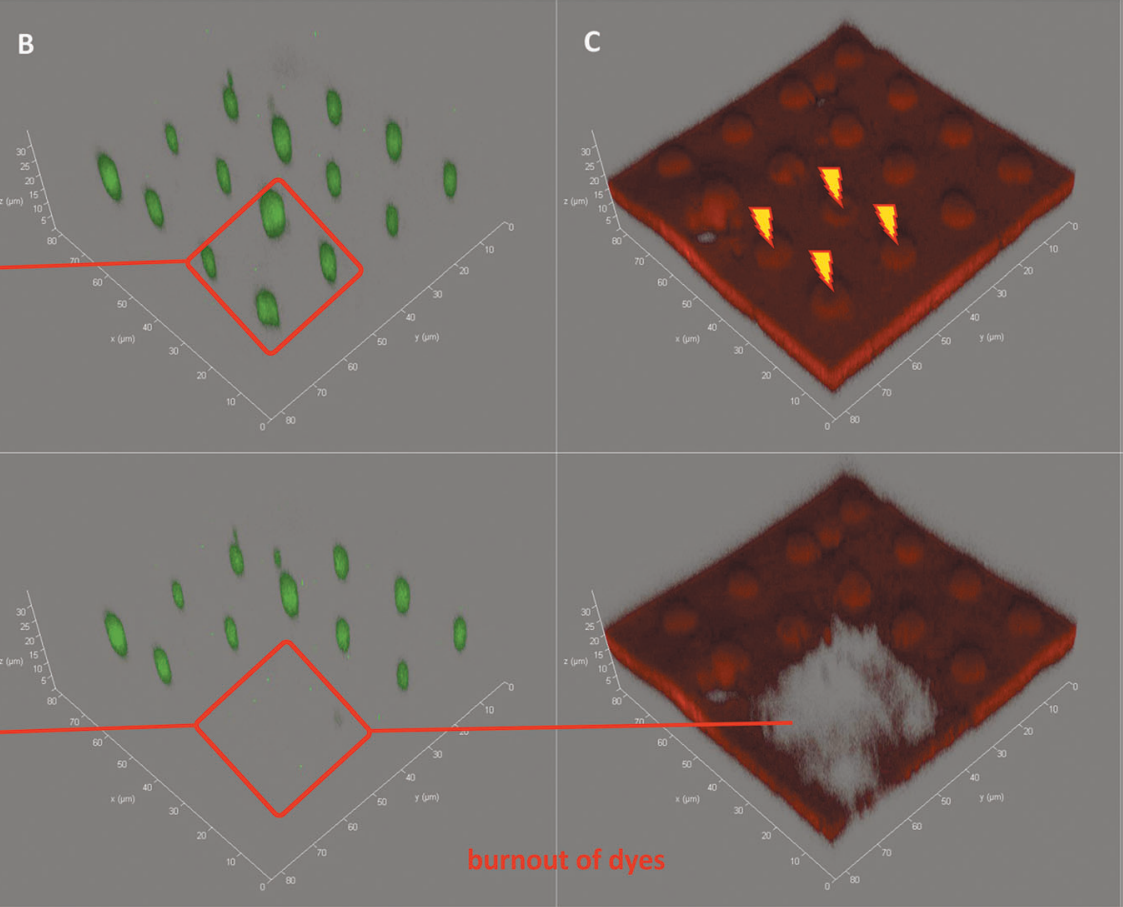

$785 \mathrm{~nm}$ laser exposure

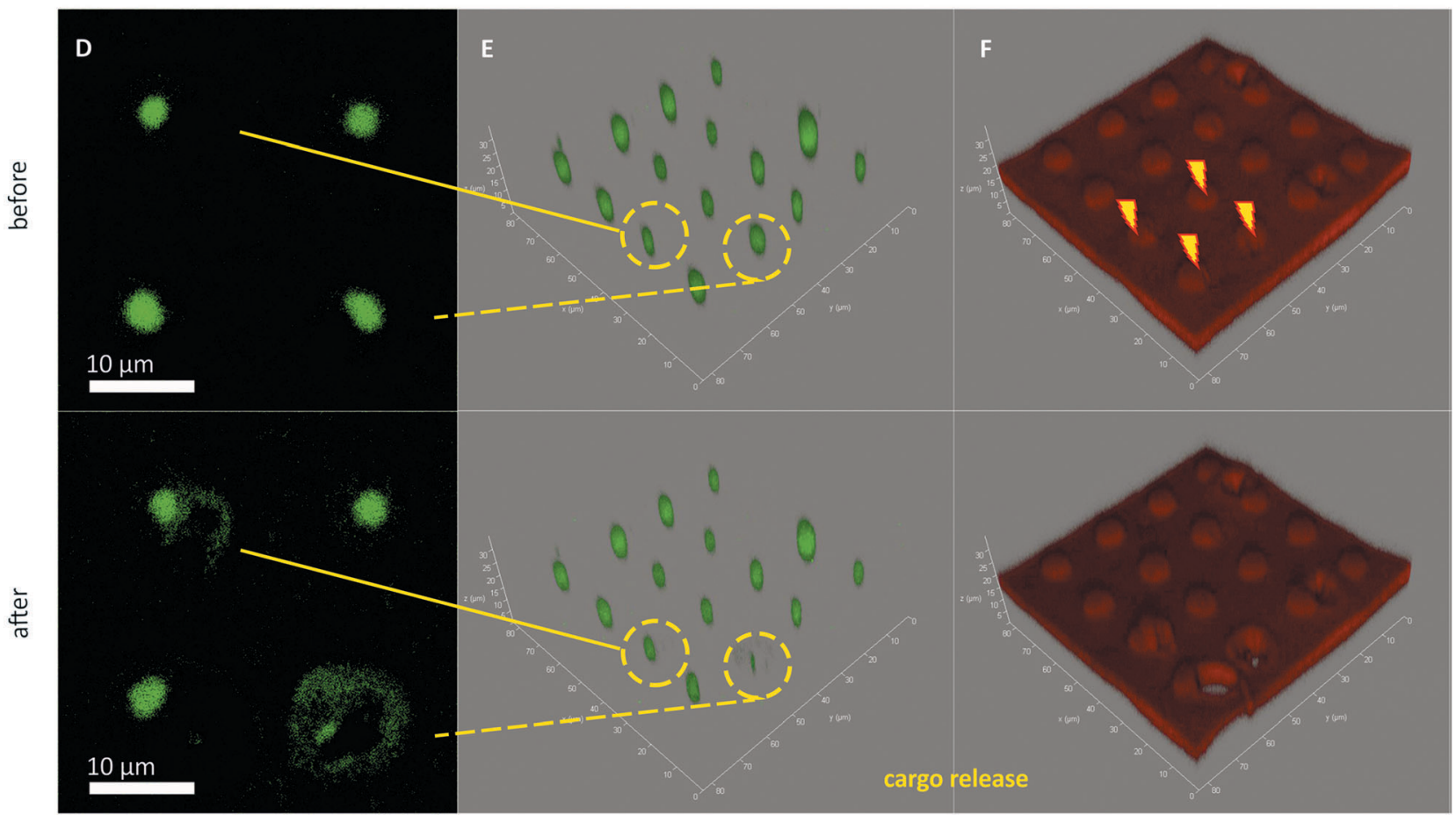

Fig. 5 Confocal images of the MCA with CDAs before and after exposure to the $532 \mathrm{~nm}(\mathrm{~A}-2 \mathrm{D} ; \mathrm{B}$ and $\mathrm{C}-3 \mathrm{D})$ and $785 \mathrm{~nm}$ lasers (D-2D; E and F - 3D). The microchambers are loaded with FITC-dextran, and PLA is labeled with Nile red. 
1 months. $^{65}$ All these properties make materials based on CDs safe and multimodal. The new materials may not only be photoabsorbing and sensitive to external stimuli but may also be used for fluorescent imaging in vivo. ${ }^{61,65}$

\subsection{Controlled fluorescent dye release from microchambers with carbon dot aggregates}

In the last stage of our research, the hypothesis that the laser exposure of CDAs leads to cargo release from the microchambers but not to shell damage was tested. For this purpose, we included the hydrophobic dye Nile red in the PLA shell and encapsulated FITC-dextran in the microchambers. An MCA was placed in a $2 \%$ agarose gel to visualize slow dye release. The laser exposure was only on 4 chambers out of 16 ; the remaining microchambers served as a control for each sample (Fig. 5).

The experiment showed that the $532 \mathrm{~nm}$ laser exposure (power, $1.662 \times 10^{4} \mathrm{~W} \mathrm{~cm}^{-2}$ ) of the MCA led to a burnout of both fluorescent dyes in the microchambers and in the carrier shell (Fig. 5A-C). In this case, dye photobleaching can be mistaken for cargo release. This result confirmed the necessity of shell labeling for more accurate detection of fluorescent cargo release.

At the same time, the use of the $785 \mathrm{~nm}$ laser (power, $0.804 \times$ $10^{4} \mathrm{~W} \mathrm{~cm}^{-2}$ ) led to a stable cargo release, as shown in Fig. 5D-F. It is worth noticing that the cargo release was detected only in 2 out of 4 microchambers that were under laser exposure owing to the lack of large CDAs in the closed microchambers. We noticed very local damage to the shells of 3 chambers (Fig. 5F) and slow diffusion of the fluorescent load (Fig. 5D and E) in 2 microchambers. This indicates that the change in the shell morphology does not always accompany cargo release. We did not observe the directional release of the fluorescent load, as was shown earlier for giant polyelectrolyte capsules containing gold nanoparticles. ${ }^{66}$ This is most likely due to the small size of the microchambers.

\section{Conclusions}

CDs can be as efficient as GNRs when used in PLA MCAs for controlled opening under laser exposure. Incorporation of CDs

ces rease by melting the thin PLA film of the microchamber walls upon focused laser exposure. CDs can be used for targeted release from each single chamber or one-byone, similar to GNPs, used for such purposes before. PLA MCAs can serve as external or implantable drug carriers in disease ent and in frequent or repeated drug administration. Most importantly, unlike the widely used gold nanostructures, CDs are made by a green chemistry approach. The concept of use of CDs is applicable to other polymeric vehicles in which light triggers the deployment of cargo. The triggering can be implemented in a non-invasive or minimally invasive way. Similar to the GNP approach, the CD approach has the potential for cellular engineering, in which a local release of chemicals is needed to stimulate individual cells.

Thus, CDs are more affordable and biodegradable than gold nanostructures and, therefore, they can be used to advantage as photoabsorption agents.

\section{Conflicts of interest}

There are no conflicts to declare.

\section{Acknowledgements}

This work was supported by the Russian Science Foundation (project no. 16-13-10195).

\section{References}

1 W. Gao, S. Emaminejad, H. Y. Y. Nyein, S. Challa, K. Chen, A. Peck, H. M. Fahad, H. Ota, H. Shiraki, D. Kiriya, D.H. Lien, G. A. Brooks, R. W. Davis and A. Javey, Nature, 2016, 529, 509-514.

2 M. V. Kiryukhin, Curr. Opin. Pharmacol., 2018, 18, 69-75.

3 M. N. Antipina, M. V. Kiryukhin, A. G. Skirtach and G. B. Sukhorukov, Int. Mater. Rev., 2014, 59, 224-244.

4 M. V. Kiryukhin, S. M. Man, A. Tonoyan, H. Y. Low and G. B. Sukhorukov, Langmuir, 2012, 28, 5678-5686.

5 M. V. Kiryukhin, S. R. Gorelik, S. M. Man, G. S. Subramanian, M. N. Antipina, H. Y. Low and G. B. Sukhorukov, Macromol. Rapid Commun., 2013, 34, 87-93.

6 M. V. Kiryukhin, H. H. Lau, S. Hong, C. Teh and V. Korzh, Talanta, DOI: 10.1016/j.talanta.2018.01.085.

7 M. Gai, J. Frueh, V. L. Kudryavtseva, A. M. Yashchenok and G. B. Sukhorukov, ACS Appl. Mater. Interfaces, 2017, 9, 16536-16545.

8 M. Gai, M. A. Kurochkin, D. Li, B. N. Khlebtsov, L. Dong, N. Tarakina, R. Poston, D. J. Gould, J. Frueh and G. B. Sukhorukov, J. Controlled Release, 2018, 276, 84-92.

9 C. Randall, T. Leong, N. Bassik and D. Gracias, Adv. Drug Delivery Rev., 2007, 59(15), 1547-1561.

10 M. Gai, J. Frueh, T. Tao, A. V. Petrov, V. V. Petrov, E. V. Shesterikov, S. I. Tverdokhlebov and G. B. Sukhorukov, Nanoscale, 2017, 9, 7063-7070.

11 A. Ermakov, S. H. Lim, S. Gorelik, A. P. Kauling, R. V. B. de Oliveira, A. H. Castro Neto, E. Glukhovskoy, D. A. Gorin, G. B. Sukhorukov and M. V. Kiryukhin, Macromol. Rapid Commun., 2018, 1700868.

12 H. Tsuji, R. Okino, H. Daimon and K. Fujie, J. Appl. Polym. Sci., 2006, 99, 2245-2252.

13 M. Houchin and E. Topp, J. Appl. Polym. Sci., 2009, 114(5), 2848-2854.

14 H. K. Makadia and S. J. Siegel, Polymers, 2011, 3, 1377-1397.

15 B. P. Timko, T. Dvir and D. S. Kohane, Adv. Mater., 2010, 22, 4925-4943.

16 M. Banchelli, S. Nappini, C. Montis, M. Bonini, P. Canton, D. Berti and P. Baglioni, Phys. Chem. Chem. Phys., 2014, 16, 10023.

17 K. Hayashi, K. Ono, H. Suzuki, M. Sawada, M. Moriya, W. Sakamoto and T. Yogo, ACS Appl. Mater. Interfaces, 2010, 2, 1903-1911.

18 D. Gourevich, O. Dogadkinac, A. Volovickac, L. Wangc, J. Gnaimb, S. Cochranc and A. Melzer, J. Controlled Release, 2013, 170(3), 316-324. 
119 H. Epstein-Barash, G. Orbey, B. E. Polat, R. H. Ewoldt, J. Feshitan, R. Langer, M. A. Borden and D. S. Kohane, Biomaterials, 2010, 31, 5208-5217.

20 D. A. Gorin, D. G. Shchukin, A. I. Mikhailov, K. Köhler, S. A. Sergeev, S. A. Portnov, I. V. Taranov, V. V. Kislov and G. B. Sukhorukov, Tech. Phys. Lett., 2006, 32, 70-72.

21 S. Huang, J. Liu, Q. He, H. Chen, J. Cui, S. Xu, Y. Zhao, C. Chen and L. Wang, Nano Res., 2015, 8, 4038-4047.

22 T. Kawano, Y. Niidome, T. Mori, Y. Katayama and T. Niidome, Bioconjugate Chem., 2009, 20, 209-212.

23 A. Wijaya, S. B. Schaffer, I. G. Pallares and K. HamadSchifferli, ACS Nano, 2009, 3, 80-86.

24 A. Agarwal, M. A. Mackey, M. A. El-Sayed and R. V. Bellamkonda, ACS Nano, 2011, 5, 4919-4926.

25 J. T. Robinson, K. Welsher, S. M. Tabakman, S. P. Sherlock, H. Wang, R. Luong and H. Dai, Nano Res., 2010, 3, 779-793.

26 A. M. Yashchenok, D. N. Bratashov, D. A. Gorin, M. V. Lomova, A. M. Pavlov, A. V. Sapelkin, B. S. Shim, G. B. Khomutov, N. A. Kotov, G. B. Sukhorukov, H. Möhwald and A. G. Skirtach, Adv. Funct. Mater., 2010, 20, 3136-3142.

27 P. Matteini, F. Tatini, L. Cavigli, S. Ottaviano, G. Ghini and R. Pini, Nanoscale, 2014, 6, 7947.

28 G. Ku, M. Zhou, S. Song, Q. Huang, J. Hazle and C. Li, ACS Nano, 2012, 6, 7489-7496.

29 X. Huang, S. Tang, X. Mu, Y. Dai, G. Chen, Z. Zhou, F. Ruan, Z. Yang and N. Zheng, Nat. Nanotechnol., 2011, 6, 28-32.

30 J. Yang, J. Choi, D. Bang, E. Kim, E.-K. Lim, H. Park, J.S. Suh, K. Lee, K.-H. Yoo, E.-K. Kim, Y.-M. Huh and S. Haam, Angew. Chem., Int. Ed., 2011, 50, 441-444.

31 Y. Liu, K. Ai, J. Liu, M. Deng, Y. He and L. Lu, Adv. Mater., 2013, 25, 1353-1359.

32 B. Radt, T. A. Smith and F. Caruso, Adv. Mater., 2004, 16, 2184-2189.

33 S. Singh, J. Nanosci. Nanotechnol., 2010, 10, 7906-7918.

36 E. Boisselier and D. Astruc, Chem. Soc. Rev., 2009, 38, 1759.

37 A. Kunzmann, B. Andersson, T. Thurnherr, H. Krug, A. Scheynius and B. Fadeel, Biochim. Biophys. Acta, Gen. Subj., 2011, 1810, 361-373.

38 X. D. Zhang, H. Y. Wu, D. Wu, Y. Y. Wang, J. H. Chang, Z. Bin Zhai, A. M. Meng, P. X. Liu, L. A. Zhang and F. Y. Fan, Int. J. Nanomed., 2010, 5, 771-781.

39 R. A. Sperling, P. Rivera Gil, F. Zhang, M. Zanella and W. J. Parak, Chem. Soc. Rev., 2008, 37, 1896.

40 E. E. Connor, J. Mwamuka, A. Gole, C. J. Murphy and M. D. Wyatt, Small, 2005, 1, 325-327.

41 J.-H. Park, L. Gu, G. von Maltzahn, E. Ruoslahti, S. N. Bhatia and M. J. Sailor, Nat. Mater., 2009, 8, 331-336.
42 X. Song, Q. Chen and Z. Liu, Nano Res., 2015, 8(2), 340-354.

43 W. Miao, H. Kim, V. Gujrati, J. Y. Kim, H. Jon, Y. Lee, M. Choi, J. Kim, S. Lee, D. Y. Lee, S. Kang and S. Jon, Theranostics, 2016, 6, 2367-2379.

44 C. Lee, W. Kwon, S. Beack, D. Lee, Y. Park, H. Kim, S. K. Hahn, S.-W. Rhee and C. Kim, Theranostics, 2016, 6, 2196-2208.

45 B. Nikoobakht and M. A. El-Sayed, Chem. Mater., 2003, 15, 1957-1962.

46 B. Khlebtsov, V. Khanadeev, T. Pylaev and N. Khlebtsov, J. Phys. Chem. C, 2011, 115, 6317-6323.

47 A. A. Kokorina, E. S. Prikhozhdenko, N. V. Tarakina, A. V. Sapelkin, G. B. Sukhorukov and I. Y. Goryacheva, Carbon, 2018, 127, 541-547.

48 F. Madzharova, Z. Heiner, J. Simke, S. Selve and J. Kneipp, J. Phys. Chem. C, 2018, 122, 2931-2940.

49 J. Midelet, A. H. El-Sagheer, T. Brown, A. G. Kanaras and M. H. V. Werts, Part. Part. Syst. Charact., 2017, 34, 1700095.

50 A. G. Skirtach, C. Déjugnat, D. Braun, A. S. Susha, A. L. Rogach and G. B. Sukhorukov, J. Phys. Chem. C, 2007, 111, 555-564.

51 W. Lauterborn and R. Ebeling, Appl. Phys. Lett., 1977, 31(10), 663-664.

52 O. Baghdassarian, B. Tabbert and G. A. Williams, Phys. Rev. Lett., 1999, 83, 2437-2440.

53 V. Kotaidis and A. Plech, Appl. Phys. Lett., 2005, 87(21), 213102.

54 D. Lapotko, Cancers, 2011, 3, 802-840.

55 S. Link, C. Burda, B. Nikoobakht and M. A. El-Sayed, J. Phys. Chem. B, 2000, 104, 6152-6163.

56 S. Link and M. A. El-Sayed, J. Chem. Phys., 2001, 114(5), 2362-2368.

57 M. Gordel, J. Olesiak-Banska, K. Matczyszyn, C. Nogues, M. Buckle and M. Samoc, Phys. Chem. Chem. Phys., 2014, 16, 71-78.

58 A. S. Angelatos, B. Radt and F. Caruso, J. Phys. Chem. B, 2005, 109, 3071-3076.

59 X. Yuan, K. Fischer and W. Schartle, Langmuir, 21, 9374-9380.

60 M. Giersig and P. Mulvaney, J. Phys. Chem., 1993, 97, 6334-6336.

61 M. Hassan, V. G. Gomes, A. Dehghani and S. M. Ardekani, Nano Res., 2018, 11, 1-41.

62 B. De and N. Karak, RSC Adv., 2013, 3, 8286.

63 D. R. Larson, W. R. Zipfel, R. M. Williams, S. W. Clark, M. P. Bruchez, F. W. Wise and W. W. Webb, Science, 2003, 300, 1434-1436.

64 Z. Zhang, Y. Duan, Y. Yu, Z. Yan and J. Chen, J. Mater. Sci.: Mater. Med., 2015, 26(7), 213.

65 H. Tao, K. Yang, Z. Ma, J. Wan, Y. Zhang, Z. Kang and Z. Liu, Small, 2012, 8, 281-290.

66 M. F. Bédard, B. G. De Geest, H. Möhwald, G. B. Sukhorukov and A. G. Skirtach, Soft Matter, 2009, 5, 3927. 